\title{
Especie nueva de Echeveria (Crassulaceae) de Tamaulipas, México
}

\section{New species of Echeveria (Crassulaceae) from Tamaulipas, Mexico}

\author{
José Guadalupe Martínez-Ávalos ${ }^{1 *}$, Arturo Mora-Olivo ${ }^{1}$ Martin Terry ${ }^{2}$ \\ ${ }^{1}$ Instituto de Ecología Aplicada, Universidad Autónoma de Tamaulipas. 13 Blvd. López Mateos 928, Cd. Victoria 87040, Tamaulipas, México. \\ ${ }^{2}$ Department of Biology, Sul Ross State University. Alpine, Texas 79832, USA. \\ *Correspondencia:jmartin@uat.edu.mx
}

\begin{abstract}
Resumen. Se describe y propone Echeveria tamaulipana (Crassulaceae) como especie nueva para la ciencia). El nuevo taxón se asemeja a los de la serie Angulatae, especialmente E. bifida, E. walpoleana y E. strictiflora con los cuales se compara por la semejanza en su morfología. Su distribución geográfica se restringe a los cañones cercanos a Ciudad Victoria, Tamaulipas.

Palabras clave: Crassulaceae, Echeveria, Tamaulipas.
\end{abstract}

\begin{abstract}
Echeveria tamaulipana (Crassulaceae) is described and proposed as a new species. The new species is similar to those of the series Angulatae, especially E. bifida, E. walpoleana and E. strictiflora, with which it is compared because of their similarity in morphology. Its geographical distribution is restricted to canyons near Ciudad Victoria, Tamaulipas.
\end{abstract}

Key words: Crassulaceae, Echeveria, Tamaulipas.

\section{Introducción}

El género Echeveria se encuentra bien representado en el continente Americano. México es el centro de mayor diversidad y endemismo de este grupo de plantas (Meyrán y López, 2003; Thiede, 1995) que está conformado por más de 100 especies, de las cuales el $95 \%$ se restringen exclusivamente al territorio mexicano (Thiede, 1995). Una región importante por su alta concentración de especies de Crasuláceas es la sierra Madre Oriental de México. Aquí, en los últimos años, numerosos investigadores han realizado una serie de expediciones botánicas y han descrito nuevas especies de los géneros Sedum y Echeveria, (MartínezÁvalos y Mora-Olivo, 2000; McDonald, 1991; Nesom, 1988; Nesom y Turner, 1995), particularmente del estado de Tamaulipas. Walther (1972) reporta de tal entidad seis especies de Echeveria (E. mucronata Schltdl., E. rosea Lindl., E. runyonii Rose, E. semivestita Moran var. floresiana Walther, E. shaviana Walther y E. walpoleana Rose), la mayoría de ellas distribuidas a lo largo de la sierra Madre Oriental, la cual abarca parte de la zona sur y oeste del estado. La riqueza conocida de especies en la zona, marcó el inicio de un estudio florístico de esta familia en el estado de Tamaulipas y zonas aledañas, de los estados de Nuevo León y San Luis Potosí. Después de hacer una

Recibido: 21 noviembre 2007; aceptado: 20 enero 2009 revisión bibliográfica y de los ejemplares de herbario de las especies del género registradas para la zona, se observó que una especie recolectada en las cercanías de Ciudad Victoria no correspondía a ninguna de las ya conocidas, por lo que se propone como un nuevo taxón para la ciencia.

\section{Descripción}

Echeveria tamaulipana J. G. Martínez-Ávalos, A. MoraOlivo et M. Terry, n. sp.

TIPO: México. Tamaulipas: Municipio de Victoria, km 10 carretera Ciudad Victoria-Jaumave, febrero de 1994, J. G. Martínez-Ávalos 0123. Holotipo: UAT. Figuras 1 y 2.

Herba perennis, glabra, acaulis, rosula $18-20$ (-30) cm diametro, foliis numerosis (25 vel pluribus), lanceolatis, 12-17 cm longis, 2.5-4.3 cm latis, atroviridibus, lucentibus. Inflorescenia unica, 1-3 ramis, bracteis lanceolatis; flores aurantiaci, pedicellis $0.2-0.4 \mathrm{~cm}$ longis, nectariis deltoideis $0.5 \mathrm{~cm}$ longis, $0.2 \mathrm{~cm}$ latis, ochraceis.

Planta herbácea perenne, glabra, solitaria. Roseta densa, sésil en el suelo, de 18 a $20(-30) \mathrm{cm}$ de diámetro. Hojas 25 o más, ascendentes, lanceoladas, aquilladas en el ápice, de 12 a $17 \mathrm{~cm}$ de largo, 2.5 a $4.3 \mathrm{~cm}$ de ancho en la parte media y 0.2 a $0.4 \mathrm{~cm}$ de grosor, las más viejas 


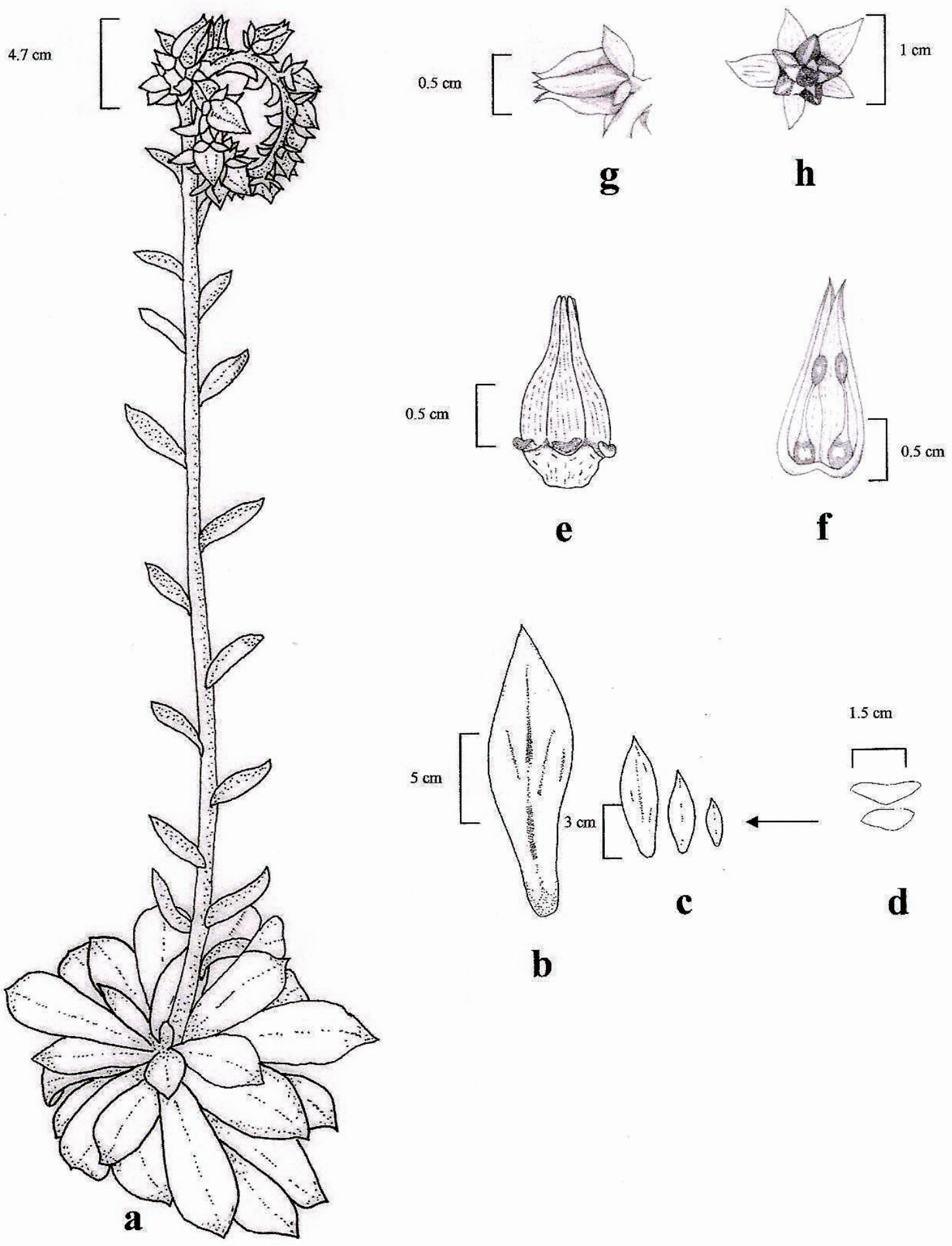

Figura. 1. Echeveria tamaulipana. J. G. Martínez-Ávalos, A. Mora-Olivo et M. Terry a, planta con inflorescencia; b, parte adaxial de la hoja; c, brácteas; d, corte transversal de una bráctea; e, nectarios; f, carpelos; $\mathbf{g}$, vista lateral de la flor; $\mathbf{h}$, vista frontal de la flor. 


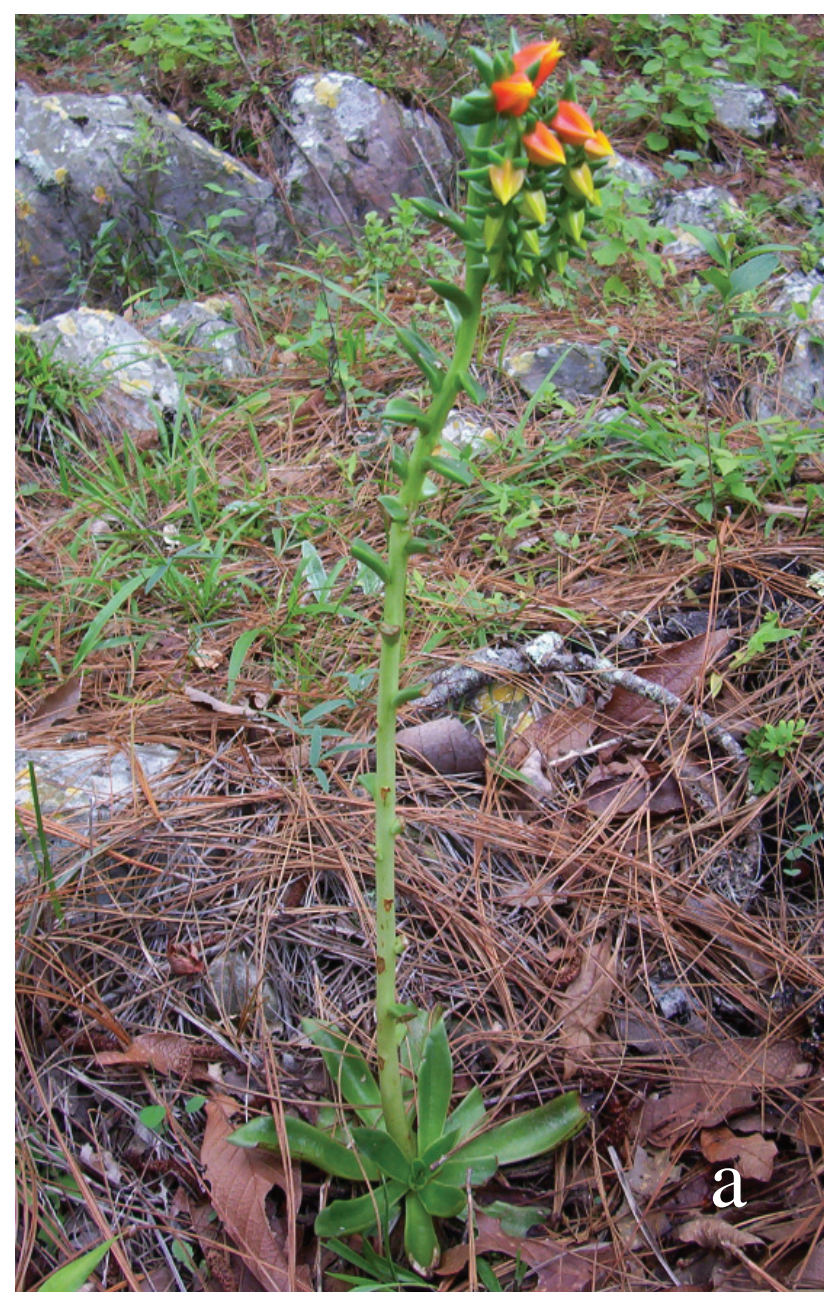

Figura 2. Echeveria tamaulipana J. G. Martínez-Ávalos, A. Mora-Olivo et. M. Terry. a, planta en su hábitat natural; b, inflorescencia; c, flor (Fotos: Gilberto Herrera).

extendidas, de color verde obscuro brillante, en ocasiones con el margen rojizo; las más jóvenes acanaladas, de color verde claro brillante, con el margen de color más claro, de 1.9 a $2.5 \mathrm{~cm}$ de largo y 0.8 a $1.2 \mathrm{~cm}$ de ancho en la parte media. Pedúnculo curvo, de 37 a $42(-50) \mathrm{cm}$ de largo, de 0.4 a $0.8 \mathrm{~cm}$ de grosor en la base, cilíndrico, de color verde claro; brácteas 25 a 31, ascendentes, alternas, las inferiores de mayor tamaño que las superiores, dispuestas en un ángulo de $35^{\circ}$, de 4.0 a $7.0 \mathrm{~cm}$ de largo por 0.9 a 1.2 $\mathrm{cm}$ de ancho y de 0.1 a $0.3 \mathrm{~cm}$ de grosor, lanceoladas; las superiores de 2.0 a $2.5 \mathrm{~cm}$ de largo, 0.5 a $0.7 \mathrm{~cm}$ de ancho y 0.2 a $0.3 \mathrm{~cm}$ de grosor, de forma obovada, algo achatadas de color verde pálido. Inflorescencia 1, con 1 a 3 ramas secundifloras, de 9.5 a $11 \mathrm{~cm}$ de longitud cada uno, de color verde claro. Flores 9 a 12 por rama; pedicelos de $0.2 \mathrm{~cm}$ a $0.4 \mathrm{~cm}$ de longitud y $0.1 \mathrm{a} 0.12 \mathrm{~cm}$ de grosor; cáliz con
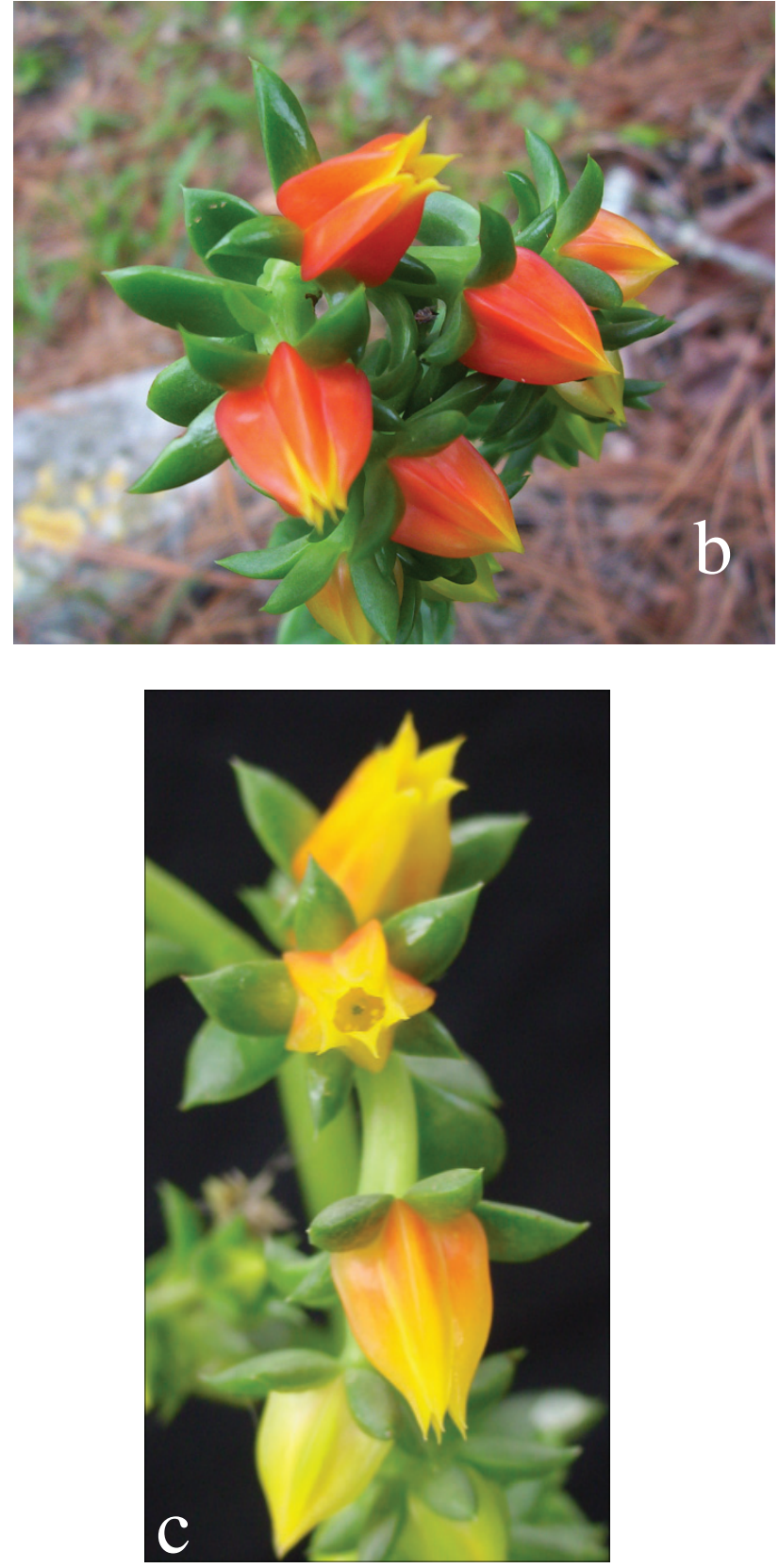

5 sépalos de color verde pálido, lanceolados, desiguales entre sí, libres, subrollizos, robustos, con el ápice agudo, el mayor de $1.3 \mathrm{~cm}$ de largo por $0.5 \mathrm{~cm}$ de ancho, el menor de $0.5 \mathrm{~cm}$ de largo y $0.3 \mathrm{~cm}$ de ancho; corola cónico-urceolada, con 5 lóbulos, de 1.0 a $1.6 \mathrm{~cm}$ de largo por 0.5 a $0.8 \mathrm{~cm}$ de ancho en la base, de forma triangular, aquillados, de color anaranjado brillante; estambres 10, 5 epipétalos de $0.6 \mathrm{~cm}$ de largo, 5 episépalos de $0.9 \mathrm{~cm}$ de largo, de color amarillo ocre; anteras de 0.2 a $0.3 \mathrm{~cm}$ de largo, de color amarillo ocre; carpelos 5, de 0.8 a $1.0 \mathrm{~cm}$ de largo por $0.3 \mathrm{a}$ 
$0.5 \mathrm{~cm}$ de ancho de color amarillo blancuzco con pequeñas manchas rojizas esparcidas. Nectarios de color amarillo cuero, ampliamente deltoides, de $0.2 \mathrm{~cm}$ de ancho, $0.5 \mathrm{~cm}$ de largo y $0.5 \mathrm{~cm}$ de grueso. Semillas alargadas de $61 \mathrm{a}$ $81 \mu \mathrm{m}$ de largo ( $71.3 \mu \mathrm{m}$ en promedio), y de 20 a $27 \mu \mathrm{m}$ de ancho $(23.6 \mu \mathrm{m}$ en promedio).

\section{Resumen taxonómico}

Fenología. Florece en los meses de abril a agosto, la inflorescencia seca perdura hasta el año siguiente.

Distribución y hábitat. Esta especie sólo se ha recolectado en los cañones de la sierra Madre Oriental, al norte, oeste y sur de Ciudad Victoria, Tamaulipas, en altitudes que van de los 800 a los $1200 \mathrm{~m}$. La vegetación corresponde a bosques de pino-encino y bosques de galería. Suele encontrarse en suelos húmedos, ricos en materia orgánica y ocasionalmente en zonas rocosas o pedregosas. Cuando la planta crece en zonas abiertas y soleadas, las hojas presentan un color verde oscuro, en ocasiones muestran manchas rojizas en los márgenes.

Etimología. El epíteto específico alude al nombre del estado donde fue recolectada.

Paratipo. México. Tamaulipas: Municipio de Victoria, Cañón del Novillo, Km. 11 Ciudad Victoria-Las Minas, 5.III. 2000, J. G. Martínez-Avalos 0870 (MEXU).

\section{Comentarios taxonómicos}

Echeveria tamaulipana se ubica en la serie Angulatae (sensu Walther, 1972), por la ausencia de tallo, hojas densamente rosuladas, la mayoría gruesas y turgentes, cóncavas en la parte superior; inflorescencia con una a 3 ramas secundifloras, pedúnculos robustos y curvos, con pocas o numerosas brácteas, éstas usualmente adpresas o ascendentes, gruesas, a menudo subrollizas; pedicelos cortos y gruesos, rara vez largos; sépalos subrollizos, más o menos anchos a mitad de la corola, profundamente hundidos en la base; corola agudamente pentagonal; nectarios largos y gruesos.

Se compara con E. bifida, E. walpoleana y E. strictiflora por la semejanza que tiene el tamaño de la roseta, hojas gruesas con el margen rojizo, inflorescencia con pedicelo robusto y pedúnculo largo y grueso, la presencia de brácteas ascendentes, lanceoladas, y el tamaño de los nectarios. Sin embargo, E. tamaulipana difiere de E. walpoleana y E. strictiflora porque presenta una roseta de mayor tamaño $(18$ a $30 \mathrm{~cm})$ que el resto de las especies, por el mayor número de hojas (25 o más), de forma lanceolada y más anchas (2.5 a $4.3 \mathrm{~cm})$, de color verde oscuro brillante; con una inflorescencia única, con 1 a 3 ramas, cada una con 9 a 12 flores de color anaranjado brillante con pedicelo grueso $(0.1$ a $0.12 \mathrm{~cm})$ y largo $(0.2$ a $0.4 \mathrm{~cm})$; pedúnculo curvo, grueso $(0.8 \mathrm{~cm})$ de color verde pálido; brácteas lanceoladas; ligeramente anchas $(1.2 \mathrm{~cm})$; cáliz ligeramente más largo $(1.3 \mathrm{~cm})$ y ancho $(0.5 \mathrm{~cm})$, muy robusto y agudo, pedicelo de mayor longitud $(0.4 \mathrm{~cm})$ que el resto de las especies. Nectarios de color amarillo cuero, ligeramente más largos y gruesos $(0.5 \mathrm{~cm})$ que los de E. bifida pero de igual tamaño que los de E. walpoleana y E. strictiflora.

Difiere de estas especies por el tamaño, forma y color de las hojas y brácteas, la presencia de pedicelos relativamente largos, así como por los carpelos gruesos y robustos. Además, diferencias notorias en la forma y tamaño de nectarios y estambres (Cuadro 1).

Adicionalmente, Echeveria tamaulipana se compara con el resto de las especies de la serie Angulatae (sensu Walther, 1972) para cotejar algunas semejanzas del tallo y hojas, así como de las partes que conforman la inflorescencia y las flores. Este grupo, que incluye a $E$. humilis Rose, E. angustifolia Walther, E. lutea Rose, E. lutea Rose var. fuscata Walter, E. tenuis Rose, E. heterosepala Rose, se diferencia de E. tamaulipana por presentar inflorescencia simple, flores pediceladas o con un pedicelo corto o en ocasiones ausente. Asimismo, $E$. tamaulipana difiere de E. humilis en las hojas, que en esta última son más delgadas, con menos de $2.2 \mathrm{~cm}$ de grosor. Es diferente de E. angustifolia en las hojas, que en ésta son muy angostas, oblanceoladas, de aproximadamente $4 \mathrm{~cm}$ de longitud y $0.9 \mathrm{~cm}$ de grosor. Se diferencia fácilmente de E. lutea, que se caracteriza por el color amarillo claro de su corola, la presencia de hojas cóncavas fuertemente curvadas en la punta y la presencia de sépalos articulados. De igual forma, E. lutea var. lutea y E. lutea var. fuscata difieren de E. tamaulipana, porque estas variedades presentan hojas de menor tamaño $(7 \mathrm{a} 11 \mathrm{~cm} \mathrm{y} 5 \mathrm{~cm}$ de longitud) y de color café verdoso, cuentan además con una inflorescencia reducida (35 y $75 \mathrm{~cm}$ de alto). Por último, E. tenuis es diferente de E. tamaulipana, porque presenta hojas muy gruesas, cortas (6 $\mathrm{cm}$ de longitud), de forma acintada y cóncavas, y flores rojizas o verdosas, nunca amarillas.

En cuanto a la distribución geográfica de las especies anteriores, y de acuerdo con los registros de Walther (1972), Thiede (1995) y Meyrán y López (2003), relacionados con la fitogeografía cuantitativa, riqueza de especies y evolución de las crasuláceas de América y de México, ninguna de las especies comparte el hábitat con E. tamaulipana (Fig. 3). La única especie que se encuentra asociada a $E$. tamaulipana es E. shaviana, especie muy abundante en la zona pero perteneciente a la serie Pruinosae. 
Cuadro 1. Comparación de algunas características morfológicas de Echeveria tamaulipana, E. bifida Schltdl., E. walpoleana Rose y E. strictiflora A. Gray

\begin{tabular}{|c|c|c|c|c|}
\hline & E. tamaulipana & E. bifida & E. walpoleana & E. strictiflora \\
\hline Tallo & ausente & usualmente corto & muy corto o ausente & muy corto o ausente \\
\hline \multicolumn{5}{|l|}{ Roseta } \\
\hline Diámetro $(\mathrm{cm})$ & 18 a $20(-30)$ & 8 a 24 & 10 a 18 & 14 a 18 \\
\hline $\mathrm{N}^{\circ}$ hojas & 25 o más & 15 & 20 & 20 \\
\hline Color & $\begin{array}{l}\text { verde claro a obscuro } \\
\text { brillante }\end{array}$ & verde opaco & verde azulado opaco & verde opaco \\
\hline \multicolumn{5}{|l|}{ Hojas } \\
\hline Forma & lanceoladas & rómbicas a oblanceoladas & obovadas a lanceoladas & romboide-oblanceoladas \\
\hline Color & verde obscuro brillante & verde opaco & verde opaco & grisáceas a glaucas \\
\hline Largo $(\mathrm{cm})$ & 12 a 17 & 4 a 12 & 5 a 9 & 7 a 9 \\
\hline Ancho $(\mathrm{cm})$ & 2.5 a 4.3 & 1 a 2.5 & 2 & 1.5 a 2 \\
\hline Grueso $(\mathrm{cm})$ & 0.2 a 0.4 & 0.4 & 0.4 & 0.3 \\
\hline Inflorescencia & $\begin{array}{c}\text { 1, con } 1 \text { a } 3 \text { ramas, con } \\
9 \text { a } 12 \text { flores de color } \\
\text { anaranjado brillante, con } \\
\text { un pedicelo de } 0.2 \text { a } 0.4 \\
\text { cm de largo. }\end{array}$ & $\begin{array}{c}1 \text { a } 3, \text { con } 1 \text { a } 2 \text { ramas, } \\
\text { con } 10 \text { a } 14 \text { flores, de } \\
\text { color anaranjado fuerte, } \\
\text { con un pedicelo de } 0.2 \text { a } \\
0.3 \mathrm{~cm} \text { de largo. }\end{array}$ & $\begin{array}{c}1 \text { o } 2 \text {, con } 2(-5) \text { ramas } \\
\text { secundifloras, con } 8 \\
\text { a } 15 \text { flores de color } \\
\text { rosado begonia en la } \\
\text { base y rojo durazno } \\
\text { en la parte superior, } \\
\text { pedicelo menor a } 0.1 \\
\text { cm de largo. }\end{array}$ & $\begin{array}{c}1 \text { a } 3, \text { simple, secundiflora, } \\
\text { con } 10 \text { a } 15 \text { flores de color } \\
\text { rosado begonia en la base y } \\
\text { rojo durazno en la parte } \\
\text { superior, pedicelo de } \\
0.1 \mathrm{~cm} \text { de largo. }\end{array}$ \\
\hline Pedúnculo & curvo & erecto & erecto & erecto \\
\hline Color & verde claro & verde opaco & verde opaco & verde opaco \\
\hline Largo $(\mathrm{cm})$ & $37-42(-50)$ & 40 & 90 & 20 \\
\hline Grueso $(\mathrm{cm})$ & $0.4-0.8$ & 0.4 & 0.6 & 0.6 \\
\hline \multicolumn{5}{|l|}{ Brácteas } \\
\hline Forma & lanceoladas & oblongo-oblanceoladas & obovadas a oblongas & oblongo-ovadas \\
\hline Largo $(\mathrm{cm})$ & $4-7$ & $2-8$ & 2 & $2-3$ \\
\hline Ancho $(\mathrm{cm})$ & $0.9-1.2$ & $0.6-1$ & & \\
\hline Grueso $(\mathrm{cm})$ & $0.1-0.3$ & 0.3 & & \\
\hline Cáliz & $\begin{array}{l}5 \text { sépalos desiguales, } \\
\text { lanceolados, de color } \\
\text { verde pálido, el mayor } \\
\text { de } 1.3 \mathrm{~cm} \text { de largo, } \\
0.5 \mathrm{~cm} \text { de ancho, } \\
\text { subrollizos, robustos. }\end{array}$ & $\begin{array}{l}\text { desiguales, deltoide- } \\
\text { lanceolados, de color } \\
\text { verde opacos, de } 1 \mathrm{~cm} \text { de } \\
\text { largo, } 0.4 \mathrm{~cm} \text { de ancho, } \\
\text { rollizos a semirrollizos. }\end{array}$ & $\begin{array}{c}\text { Sépalos lanceolados, } \\
\text { desiguales, de color } \\
\text { verde opaco, el mayor } \\
\text { hasta de } 1 \mathrm{~cm} \text { de largo, } \\
0.4 \mathrm{~cm} \text { de ancho, } \\
\text { agudos. }\end{array}$ & $\begin{array}{l}\text { Sépalos deltoide-oblongos, } \\
\text { desiguales, color verde } \\
\text { opaco, el mayor de } 1.3 \mathrm{~cm} \\
\text { de largo, } 0.3 \mathrm{~cm} \text { de ancho, } \\
\text { agudos. }\end{array}$ \\
\hline \multicolumn{5}{|l|}{ Nectarios } \\
\hline Color & amarillo cuero & amarillo & amarillo blancuzco & amarillo cuero \\
\hline Forma & deltoides & romboide-oblongos & triangular-reniformes & oblicuos a reniformes \\
\hline Largo $(\mathrm{cm})$ & 0.5 & 0.4 & 0.5 & 5 \\
\hline Ancho $(\mathrm{cm})$ & 0.2 & 0.3 & 0.25 & 1.5 \\
\hline Grueso $(\mathrm{cm})$ & 0.5 & 0.4 & 0.5 & 5 \\
\hline
\end{tabular}




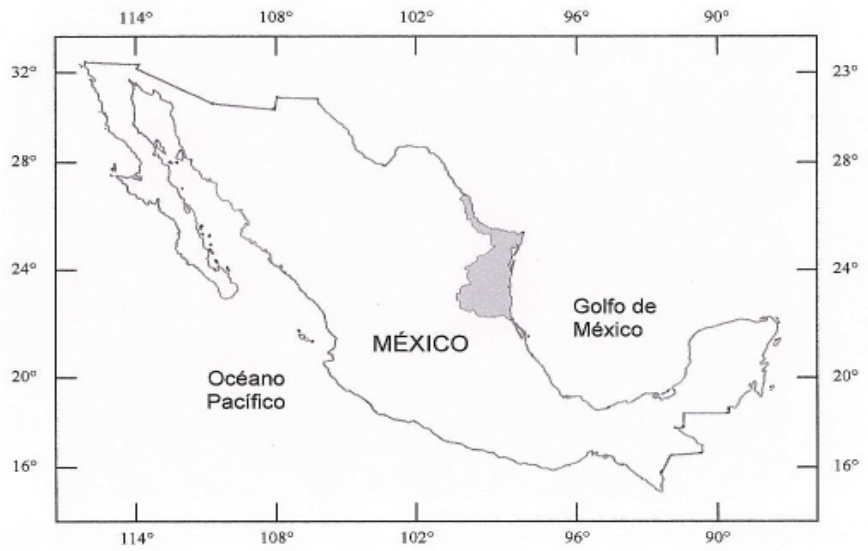

Figura. 3. Ubicación geográfica del estado de Tamaulipas y mapa de distribución de Echeveria tamaulipana $(\boldsymbol{\Delta})$.

\section{Agradecimientos}

A los revisores anónimos de esta revista; al Dr. Fernando Chiang por la revisión del artículo y por la traducción de la diagnosis al latín; al Consejo Tamaulipeco de Ciencia y Tecnología (COTACYT) por el apoyo brindado para la publicación de este trabajo. Al proyecto Fondos Sectoriales CONACYT-CONAFOR 69919.

\section{Literatura citada}

Martínez-Ávalos, J. G. y A. Mora-Olivo. 2000. Una nueva especie de Echeveria (Crassulaceae) del estado de Tamaulipas, México. Acta Botanica Mexicana 52: 43-48.

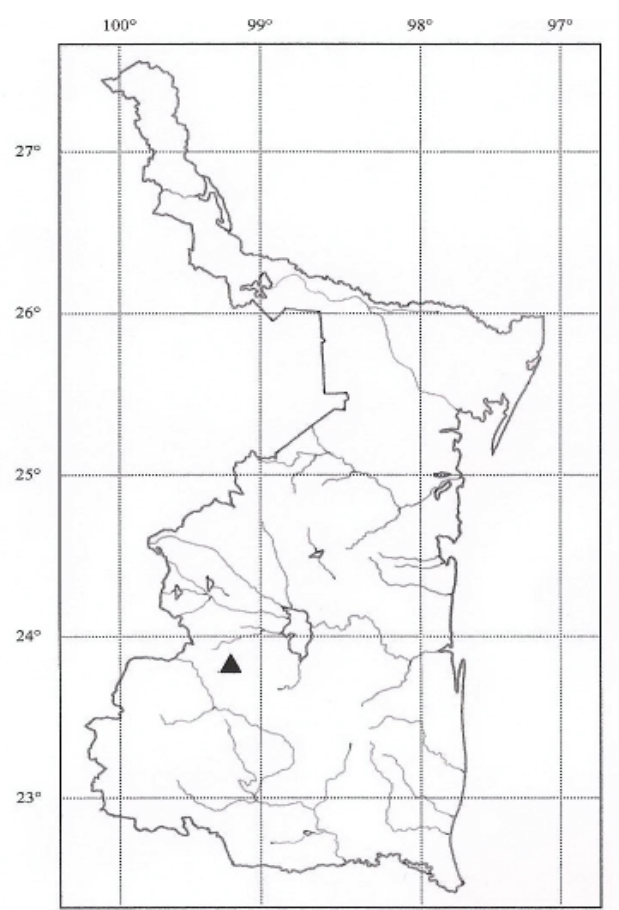

McDonald, J. A. 1991. Plantae alpinae novae mexicanae: Sedum chrysicaulum (Crassulaceae). Sida 14: 315-319.

Meyrán, J. y L. López. 2003. Las crasuláceas de México. Sociedad Mexicana de Cactología, A. C. México, D. F. 286 p.

Nesom, G. L. y B. L. Turner. 1995. Systematics of the Sedum parvum (Crassulaceae) in Northeastern Mexico and Texas. Sida 79: 257-268.

Nesom, G. L. 1988. New species of Crassulaceae from northeastern Mexico. Sida 13: 21-24.

Thied, J. 1995. Quantitative phytogeography, species richness, and evolution of American Crassulaceae. In Evolution and systematics of the Crassulaceae, Henk't Hart y Urs Eggli (eds.). Backhuys, Leiden. p. 89-123.

Walther, E. 1972. Echeveria. California Academy of Sciences, San Francisco California. 426 p. 